\title{
Dynamic Stall on a Fully Equipped Helicopter Model
}

\author{
K. Mulleners ${ }^{1}$, K. Kindler ${ }^{2}$, M. Raffel \\ German Aerospace Centre, Bunsenstraße 10, 37073 Göttingen, Germany
}

\begin{abstract}
Three-dimensional dynamic stall was observed on the rotor of a fully equipped subscale helicopter model in a wind tunnel. Results of stereoscopic particle image velocimetry measurements on the retreating blade clearly revealed the large-scale dynamic stall vortex at $50 \%$ and $60 \%$ blade radius. The small-scale shear layer vortices that constitute the large-scale dynamic stall vortex were identified and localised. Based on the spatial distribution of these smallscale vortices, the characteristic large-scale structure was found to be unexpectedly compact. Along with an apparent spanwise flexion of the dynamic stall vortex, these results suggested the rotational motion of the rotor to have a stabilising effect on the formation and convection of the dynamic stall vortex. The velocity field information available provides a basis for validating recent computational fluid dynamics results of three-dimensional dynamic stall.
\end{abstract}

Keywords: dynamic stall, helicopter, particle image velocimetry

\section{Nomenclature}

$c=$ blade reference chord length

$\hat{e}_{y} \quad=$ unit vector in $y$

$r=$ radial coordinate

$R=$ rotor blade radius

$u, v, w=$ local velocity components

$x, y, z=$ spatial coordinates

$M \quad=\quad$ reference point in $S$

$M a_{\infty}=$ free-stream Mach number

$M a_{M R}=$ rotor tip Mach number

$N=$ number of points in $S$

$P \quad=$ point

$S=$ two-dimensional area

$U_{\infty}=$ free stream velocity

$\alpha=$ rotor angle of attack

$\Gamma_{2}=$ scalar function

$\lambda_{2}=$ flow field operator

$\mu \quad=$ advance ratio

$\tau=$ blade twist

$\omega=$ vorticity

$\Omega \quad=$ angular velocity of the main rotor

$\psi \quad=$ rotor azimuth angle

${ }^{1}$ Current address: Institute of Turbomachinery and Fluid Dynamics, Leibniz Universität Hannover, Appelstrasse 9, 30167 Hannover, Germany

${ }^{2}$ Current address: Max Planck Institute for Marine Microbiology, Celsiusstr. 1, 28359 Bremen, Germany

Preprint submitted to Aerospace Science and Technology

\section{Introduction}

Dynamic stall (DS) on an airfoil comprises a series of complex aerodynamic phenomena in response to an unsteady change of the angle of attack $[1,2,3]$. It is accompanied by a lift overshoot and delayed massive flow separation, with respect to static stall, which is caused by the formation and convection of the large-scale dynamic stall vortex.

Hithereto, experimental investigations of DS have been limited to two-dimensional flows over oscillating airfoils [4, 2, 5, 6] and DS on finite wings of small aspect ratio [7, 8]. Computational fluid dynamics (CFD) investigations, on the other hand, have been constrained by challenges in turbulence modelling and limited computational performance. Only recently, CFD studies of three-dimensional DS on large aspect ratio planforms, i.e. generic helicopter blades, have become available [9] and clearly demonstrated the impact of three-dimensionality on modelling DS in the rotor operating environment. Finite wing and rotational effects as well as blade lag motion should be considered for a comprehensive understanding of DS on retreating helicopter rotor blades.

Three-dimensional DS on finite wings is essentially characterised by the interaction of the tip vortices and the DS vortex which results in the emergence of a localised omega-shaped separation region in the midspan section of the wing $[10,11,12,13]$. The DS vor-

May 23, 2011 
tex arches away from the wing surface as a result of its spanwise non-uniform emergence and development. Near the wing tips, the DS vortex is pinned to the surface and is weaker than in the midspan section, where the effective angle of attack is increased due to the downwash induced by the wing tip vortices. Whereas the dominant central part can be presumed quasi-twodimensional, the three-dimensionality and complexity of the flow field increases towards the wing tip due to the influence of the tip vortex and the accompanied arching of the DS vortex.

In the rotor environment, the concurrence of yaw and rotation leads to an additional variation of the effective angles of incidence along the blade span. The influence of the yaw angle and the rotation of a large aspect ratio blade on DS were investigated numerically by Spentzos et al. [9] revealing an asymmetrically arched DS vortex which is skewed towards the outboard side of the blade. For the case of an isolated rotor under DS conditions, recent combined experimental and numerical results indicate the presence of an outboard pointing radial jet in the DS vortex core in response to centrifugal forces $[14,15]$. The jet eventually breaks up into discrete structures due to an apparent shear layer instability, while the peak radial velocity decays towards the blade tip.

Further advancement in modelling and predicting DS on helicopter rotors hinges on the systematic rationalisation of the three-dimensionality including rotational effects. In the framework of the European Union funded project Generation of Advanced Helicopter Experimental Aerodynamic Database for CFD code validation (GoAHEAD) a fully equipped model of a generic, medium size transport helicopter was investigated in the Large Low-speed Facility (LLF) of the German-Dutch Wind Tunnels (DNW) [16, 18, 17]. In particular, phase-locked, high-resolution, threecomponent velocity field measurements of the DS vortex were conducted directly within the rotating system. Thereby, a first quantitative insight into DS on helicopter rotors was achieved. Based on these results and the lessons learned from the experiments, a future research strategy pinpointing the essential pending fluid dynamical problems is outlined.

Figure 1: Schematic representation of the experimental configuration and the positions of the measurement planes on the retreating blade at $\psi=272.3^{\circ}$. 


\section{Experimental Methods}

The helicopter model consisted of a scaled NH90 fuselage including all control surfaces, main and tail rotor. A $40 \%$ Mach-scaled ONERA 7AD main rotor with parabolic tips including a rotor hub was used. The blade radius was $R=2.1 \mathrm{~m}$ with $c=0.14 \mathrm{~m}$ average chord length (corresponding to an aspect ratio of $Æ=15)$. The main rotor had a build in inclination angle of $-5^{\circ}$ with respect to the fuselage and the blades featured $\tau=-8.3^{\circ} / R$ linear twist. The model was positioned in the centre of the $8 \mathrm{~m} \times 6 \mathrm{~m}$ closed test section of the DNW LLF.

Since the test conditions for DS were considered high-risk for the integrity of the model, stall onset was approached by varying the trim parameters at freestream and rotor tip Mach numbers of $M a_{\infty}=0.259$ and $M a_{M R}=0.617$, at $\alpha=-3.95^{\circ}$ angle of attack. The angular velocity of the main rotor was $\Omega=101.1 \mathrm{rad} / \mathrm{s}$ corresponding to an advance ratio $\mu=U_{\infty} /(\Omega R)=0.42$ with $U_{\infty}=88.3 \mathrm{~m} / \mathrm{s}$. Approaching the power, vibra- tion, and actuator force limits, a pitch link force spike in the range $\psi=180^{\circ}$ to $270^{\circ}$ was encountered indicating stall inception $\left(\psi=0^{\circ}\right.$ denotes the rear/downstream position of the blade).

Stereoscopic particle image velocimetry (PIV) data were acquired at three radial positions, $r / R=0.5,0.6$, and 0.9 (figure 1) on the retreating blade at $\psi=270^{\circ}$ to $276^{\circ}$ using standard procedures [19]. Illumination was provided by a double-cavity Nd:YAG laser with a pulse energy of $2 \times 280 \mathrm{~mJ}$ fanned out into a light sheet and directed into the test section from the ceiling of the wind tunnel. In order to reduce light reflections on the upper surface of the blade, the light sheet access was located far downstream from the model to achieve an approximately tangential impingement on the surface of the blade. Due to the limited optical access, the observation regions on the blade were inclined by $22^{\circ}, 21^{\circ}$, and $3^{\circ}$ at $r / R=0.5,0.6$, and 0.9 with respect to chord. Series of $N=100$ images were acquired through lateral windows in the wind tunnel wall using a pair of high-

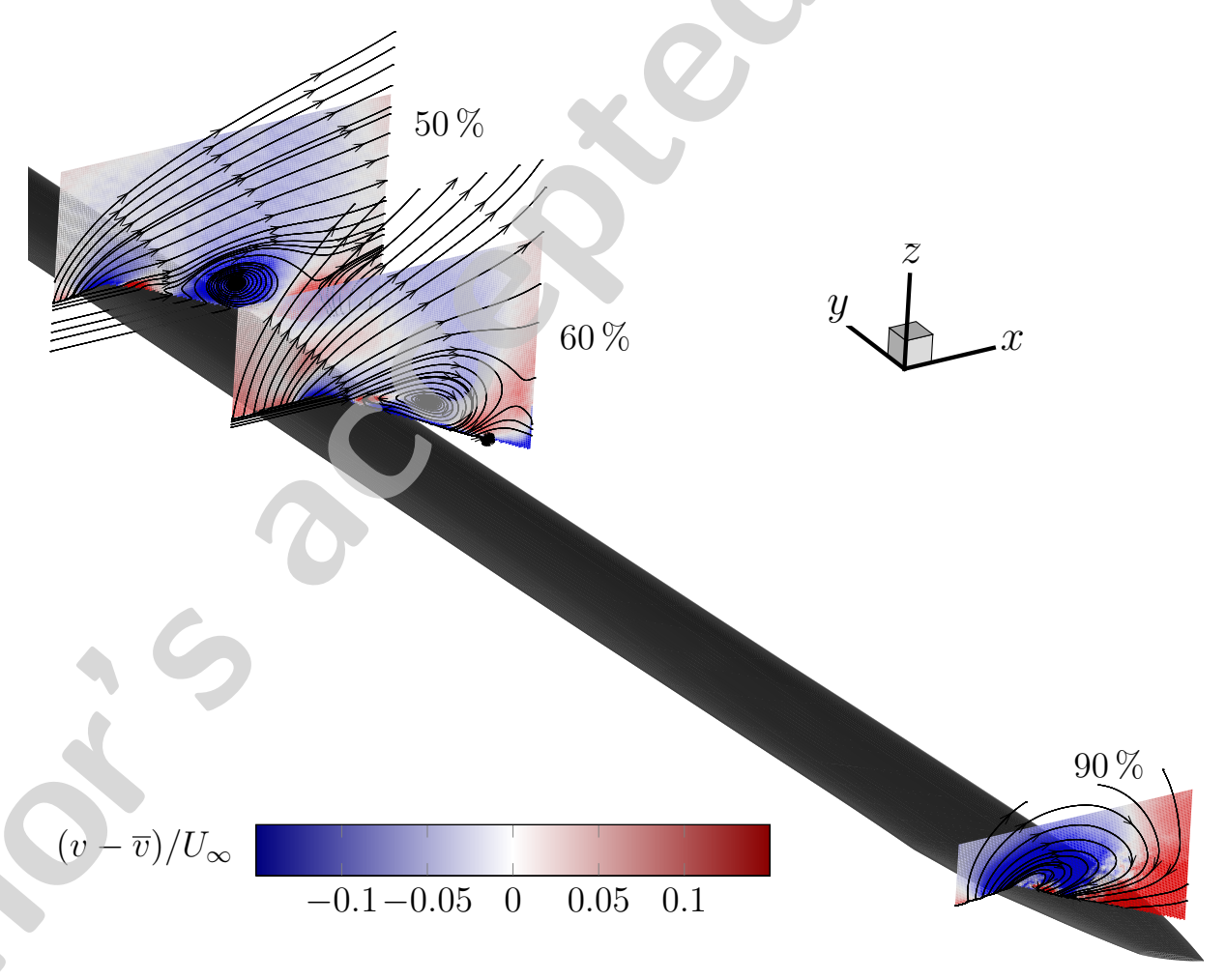

Figure 2: The footprint of dynamic stall in the three measurement planes at $r / R=0.5,0.6$, and 0.9 , at $\psi=272.3^{\circ}$ in azimuth; the scaled, phase averaged out-of-plane velocity component $v$ is colour-coded while the in-plane components $u, w$ are depicted as stream lines. 
resolution pco. 2000 cameras fitted with $200 \mathrm{~mm}$ lenses. Image acquisition was pulse-triggered by the main rotor with the cameras operating at a multiple of the rotor frequency. The fields of view were $0.23 \mathrm{~m} \times 0.22 \mathrm{~m}$ to $0.164 \mathrm{~m} \times 0.164 \mathrm{~m}$ in size corresponding to a spatial resolutions of $1.4 \mathrm{~mm}$ to $1 \mathrm{~mm}$.

\section{Results and Discussion}

\subsection{Phase averaged flow fields}

At free-stream and rotor tip Mach numbers of $M a_{\infty}=0.259$ and $M a_{M R}=0.617$, respectively, a largescale coherent DS vortex can be clearly identified at midspan $(r / R=0.5$ and 0.6$)$ whereas at the most outboard position $(r / R=0.9)$ a fully separated, strongly three-dimensional flow was observed (figure 2 ). In this region the blade tip vortex interacted with the outboard segment of the DS vortex.

The phase averaged velocity fields along with the inplane vorticity fields showed the chordwise position of the DS vortex to be unchanged between $r / R=0.5$ and 0.6 (figure 3 ). In this spatial region the DS vortex appeared to be stretched parallel to the leading edge in good agreement with recent CFD results by Spentzos [9]. However, this large-scale structure was noticeably flatter at $r / R=0.6$ than at midspan while the associated region of concentrated in-plane vorticity $\omega_{y}$ was distinctly enlarged. The radial or out-of-plane velocity component at $r / R=0.5$ was strongly negative, i.e. directed outboard, in a concentrated region covered by the DS vortex (figure 3a). The area of strong outboard swirling flow was flanked at both sides by patches of inboard directed flow, which were also observed at $r / R=0.6$ (figure $3 \mathrm{~b}$ ). The strength of the outboard directed velocity component was attenuated and the extent of the covered region was reduced conforming with previous observations of a radial jet-like flow on an isolated rotor under DS conditions $[14,15]$. In close agreement with the above cited experimental and numerical findings, a longitudinal DS vortex was observed at midspan. As a result of blade rotation, the radial velocity in the core of the large-scale structure was directed outboard. Towards the blade tip, the peak radial velocity decreased while the core of the vortex tapered.

\subsection{Substructures of the dynamic stall vortex}

Although the DS vortex appeared as a single compact vortical structure in figure 3 , it was essentially formed by the roll-up of the shear layer that emerged at the interface between the separated flow near the airfoil's surface and the free stream flow. Hence, the DS vortex was composed of a multitude of small-scale vortices generated by a primary instability of this shear layer. To study the constitution and compactness, as well as the cycle-to-cycle variability of the large-scale DS vortex, the individual small-scale vortical structures were identified and localised by means of a Galilean-invariant, Eulerian vortex axis identification procedure. The position of the centre of vortical structures in the ensemble of velocity fields was determined utilising the scalar function introduced by Graftieaux et al. [20]:

$$
\Gamma_{2}(P)=\frac{1}{N} \sum_{S} \frac{\left[P M \times\left(U_{M}-\tilde{U}_{P}\right)\right] \hat{e}_{y}}{\|P M\| \cdot\left\|U_{M}-\tilde{U}_{P}\right\|},
$$

where $N$ is the number of points in the two dimensional neighbourhood $S$ of any given point $P$ in the $x, z$ plane, $M$ lies in $S, \hat{e}_{y}$ is the unit vector in $y$ direction, and $\tilde{U}_{P}$ is the local convection velocity around $P$. The local extrema of $\Gamma_{2}$ were associated with the location of vortex centres $^{3}$.

Unlike alternative vortex identification criteria, such as vorticity concentration, $\lambda_{2}$, etc. , the $\Gamma_{2}$ function does not require the evaluation of velocity field gradients and is therefore less susceptible to experimental noise (for a comprehensive review of performance and robustness of gradient based vortex detection methods see e.g. [21, 22]).

As appears from the spatial distribution of the individual small-scale shear layer vortices extracted from the ensemble of instantaneous velocity fields at $r / R=$ 0.5 and 0.6 and their vertical and streamwise distributions (figure 4), the DS vortex was found to be strongly localised. The consequences are twofold. First, the strong confinement refers to a spatially very compact DS vortex which remains in close proximity to the airfoil's surface in contrast to two-dimensional DS [23, 24]. The rotation of the blade and the associated spanwise velocity gradient seem to have a stabilising effect on the DS vortex. Second, the cycle to cycle variations of the vortex positions were relatively small, which also indicated stabilisation of the large scale structure by the radial forcing.

\section{Conclusions and Directions for Future Experi- ments}

Dynamic stall was directly observed on the retreating blade of the rotor of a fully equipped subscale helicopter model during wind tunnel tests. The characteristic DS

\footnotetext{
${ }^{3}$ Note that $\Gamma_{2}$ is not to be confused with the circulation of a vortex.
} 

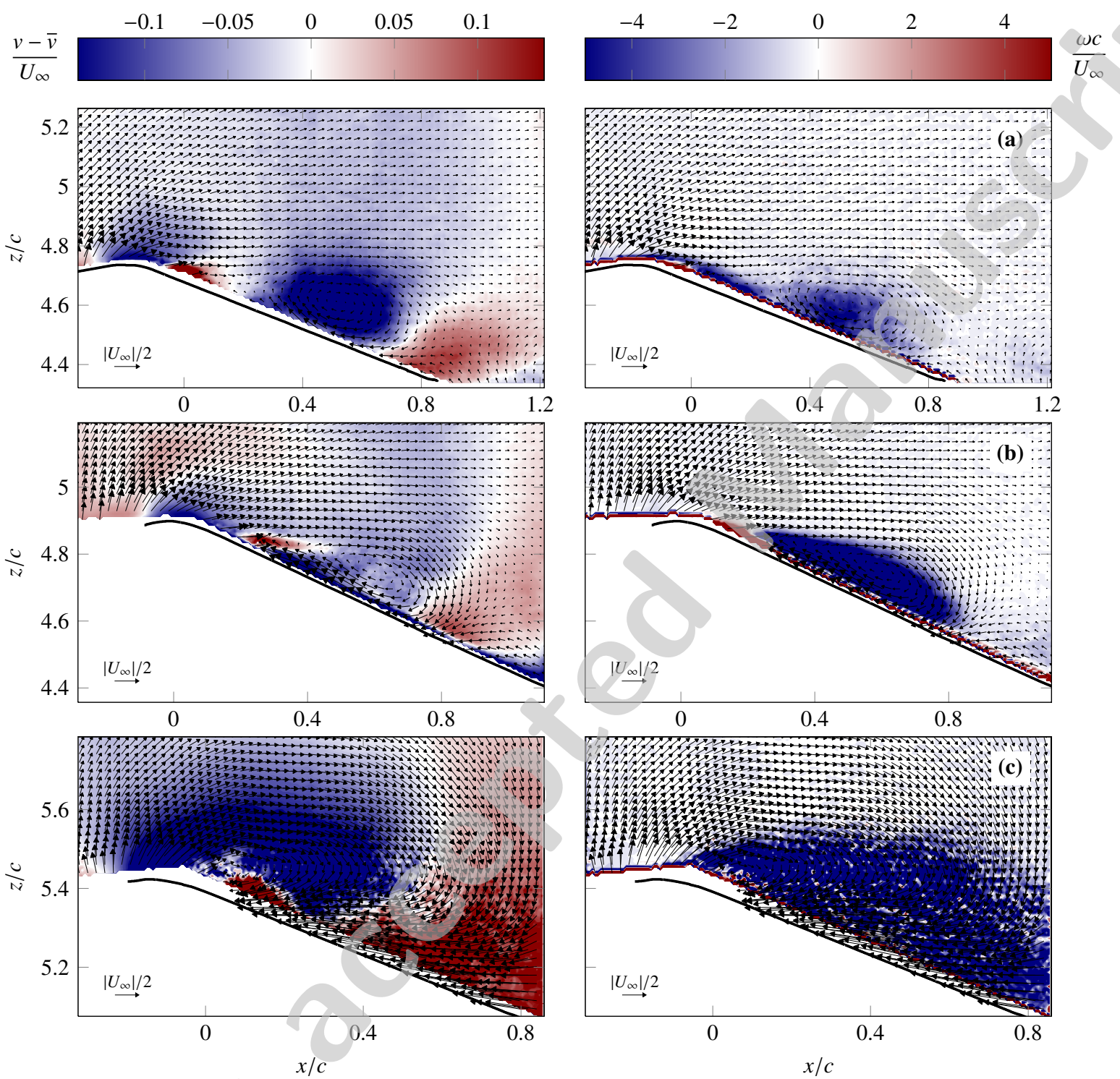

Figure 3: The scaled, phase averaged velocity (left) and the in-plane vorticity component (right) at $\psi=272.3^{\circ}$ and $r / R=0.5$ (a), 0.6 (b), and $0.9(\mathbf{c})$. The free stream velocity $U_{\infty}$ was subtracted in all vector fields while the out-of-plane component was normalised as $(v-\bar{v}) / U_{\infty}$ with $\bar{v}$ taken far from the blade surface.

vortex was identified and appeared to be stretched almost parallel to the leading edge in the region covering the measurement planes at $50 \%$ and $60 \%$ blade radius. At $r / R=0.9$, the blade tip vortex interacted with the outboard segment of the DS vortex yielding increased three-dimensionality and complexity of the flow field. Based on the identification and analysis of the individ- ual small-scale shear layer vortices composing the DS vortex, the latter was found to be spatially concentrated which was attributed to a combination of rotational and finite wing effects.

These first quantitative measurements of the DS phenomenon on a fully equipped helicopter model provide a validation database for recent CFD results. Never- 

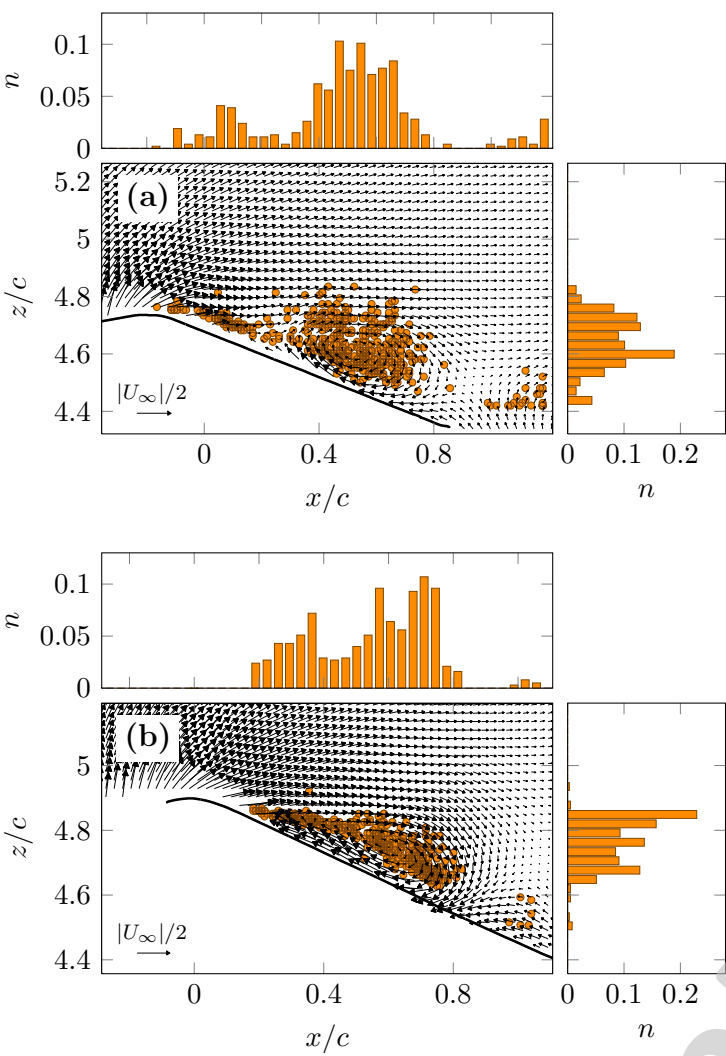

Figure 4: The in-plan velocity, the centre positions of substructures composing the DS vortex and the distribution of centre positions at $\psi=272.3^{\circ}$ for $r / R=0.5$ (a), $0.6(\mathbf{b})$

theless, additional extensive experimental efforts taking into account the rotor operating environment are required in order to advance the fundamental understanding of three-dimensional DS on helicopters.

Two successive experiments are proposed aiming at a detailed characterisation of the influence of yaw and blade rotation on the onset and development of the dynamic stall process; the spanwise development of DS vortex; and the interaction between the DS vortex and other viscous related unsteady aerodynamic phenomena, such as blade tip vortices. First, the interaction of the end segment of the DS vortex with the blade tip vortex on a finite pitching airfoil section in a wind tunnel in relation to the yaw angle should be studied. Second, combining rotation and three-dimensional effect, the ultimate experiment should provide detailed information of the whole life cycle of rotor DS by means of high fidelity PIV to improve modelling and prediction of the complex aerodynamic phenomena attributed to DS. For this purpose, it is envisaged to evoke DS on a subscale rotor in a hover facility building the image acquisition system into the rotor hub and providing a rotating laser sheet from above the rotor axis. By doing so, the continuous observation of the velocity field and the development of the characteristic DS vortex as a function of azimuthal position will be possible.

\section{Acknowledgements}

This work has been part of the European Commission funded project "Generation of Advanced Helicopter Experimental Aerodynamic Database for CFD code validation" (GoAhead). Valuable discussion and support by the project partners is greatfully acknowledged.

\section{References}

[1] L. Carr, K. McAlister, W. McCroskey, Analysis of the development of dynamic stall based on oscillating airfoil experiments, Technical Note D-8382, National Aeronautics and Space Administration, USA (1977).

[2] K. McAlister, L. Carr, W. McCroskey, Dynamic stall experiments on the NACA0012 airfoil, Technical Publication 1100, National Aeronautics and Space Administration, USA (1978).

[3] W. McCroskey, The phenomenon of dynamic stall, Technical Memorandum 81264, National Aeronautics and Space Administration, USA (1981).

[4] W. McCroskey, R. Fisher, Dynamic stall of airfoils and helicopter rotors, Advisory Group for Aerospace Research and Development R 595, Advisory Group for Aerospace Research and Development (1972).

[5] L. Carr, Progress in analysis and prediction of dynamic stall, Journal of Aircraft 25 (1) (1988) 6-17.

[6] M. Raffel, J. Kompenhans, P. Wernert, Investigation of the unsteady flow velocity field above an airfoil pitching deep dynamic stall conditions, Experiments in Fluids 19 (1995) 103-111.

[7] J. Szafruga, B. Ramaprian, LDA measurements in the threedimensional flow over an oscillating wing, in: 28th AIAA Fluid Dynamics Conference, Snowmass, CO, 29 June - 2 July (1997).

[8] F. N. Coton, R. A. M. Galbraith, An experimental study of dynamic stall on a finite wing, The Aeronautical Journal 103 (1023) (1999) 229.

[9] A. Spentzos, G. N. Barakos, K. J. Badcock, B. E. Richards, Modelling three-dimensional dynamic stall of helicopter blades using computational fluid dynamics and neural networks, Proceedings of the Institution of Mechanical Engineers Vol. 220 Part G; Journal of Aerospace Engineering (2006) 605.

[10] M. Horner, Controlled three-dimensionality in unsteady separated flows about a sinusoidally oscillating flat plate, in: Proceedings of the $28^{\text {th }}$ Aerospace Science Meeting, Reno, Nevada, USA, American Institute of Aeronautics and Astronautics paper no. 90-0689 (1990).

[11] R. Piziali, 2D and 3D oscillating wing aerodynamics for a range of angle of attack including stall, Technical Memorandum 4632, National Aeronautics and Space Administration, USA (1994).

[12] S. Schreck, H. Helin, Unsteady vortex dynamics and surface pressure topologies on a finite wing, Journal of Aircraft 31 (4) (1994) 899-907. 
[13] S. Moir, F. Coton, An examination of the dynamic stalling of two wing planforms, Aero Report 9526, Glasgow University (1995).

[14] J. DiOttavio, K. Watson, J. Cormey, N. Komerath, Discrete structures in the radial flow over a rotor blade in dynamic stall, in: Proceedings of the $26^{\text {th }}$ Applied Aerodynamics Conference, American Institute of Aeronautics and Astronautics, Honolulu, Hawaii (2008).

[15] V. Raghav, P. Richards, N. Komerath, M. Smith, An exploration of the physics of dynamic stall, in: Proceedings of American Helicopter Society Aeromechanics Specialists' Conference, American Helicopter Society, San Francisco, CA, USA (2010).

[16] K. Pahlke, The GoAHEAD project, in: Proceedings of the $33^{\text {rd }}$ European Rotorcraft Forum, Kazan, Russia (2007)

[17] M. Raffel, F. de Gregorio, K. de Groot, O. Schneider, W. Sheng, G. Gibertini, A. Seraudie, On the generation of a helicopter aerodynamics database, The Aeronautical Journal 115(1146), paper no. 3565 (2011).

[18] M. Raffel, F. de Gregorio, W. Sheng, G. Gibertini, A. Seraudie, K. de Groot, B. van der Wall, Generation of an advanced helicopter experimental aerodynamic database - The wind tunne test of the EU-project GoAHEAD, in: Proceedings of the $35^{\text {th }}$ European Rotorcraft Forum, Hamburg, Germany, (2009).

[19] M. Raffel, C. Willert, S. Wereley, J. Kompenhans, Particle Image Velocimetry, A practical guide, $2^{\text {nd }}$ edition, Springer (2007).

[20] L. Graftieaux, M. Michard, N. Grosjean, Combining piv, pod and vortex identification algorithms for the study of unsteady turbulent swirling flows, Measurement Science and Technology 12 (2001) 1422.

[21] J. Jeong, F. Hussain, On the identification of a vortex, Journal of Fluid Mechanics 285 (1995) 69-94.

[22] R. Cucitore, M. Quadrio, A. Baron, On the effectivenss and limitations of local criteria for the identification of a vortex, European Journal of Mechanics B - Fluids 18 (2) (1999) 261-282.

[23] K. Mulleners, M. Raffel, The onset of dynamic stall revisited, Experiments in Fluids (2011) in press.

[24] C. Shih, L. Lourenco, L. Van Dommelen, A. Krothapalli, Unsteady flow past an airfoil pitching at constant rate, American Institute of Aeronautics and Astronautics Journal 30 (5) (1992) 1153-1161. 